\title{
Surgical Management of a Case of Severe Coarctaton of Aorta with Biscuspic Aortic Valve
}

\author{
MN HASAN ${ }^{\mathrm{a}}$, MA SIDDIQUE ${ }^{\mathrm{b}}$, SK BANERJEE ${ }^{\mathrm{c}}$, AS ABDULLAH ${ }^{\mathrm{d}}$
}

\begin{abstract}
Summary:
Coarctation of the aorta is a congenital cardiac malformation that can go undiagnosed until old age with only hypertension as a marker of its presence because clinical signs can be subtle and overlooked if a complete physical exam is not performed. Long-term survival is exceptional in patients with untreated aortic coarctation. In this case report, we present a late diagnosis of aortic
\end{abstract}

Introduction:

Aortic coarctation is a congenital vascular lesion typically diagnosed in early life, accounting for 5 to $10 \%$ of all congenital cardiovascular malformations but may go undetected well until adulthood ${ }^{1}$. It manifests as childhood hypertension, lower extremity fatigue or weakness, diminished lower extremity pulses and or congestive heart failure. Diagnosis is usually based on clinical suspicion and physical findings. The latter include blood pressure difference between the upper and lower extremities, pulse delay and systolic murmur over the thoracic spine. Other manifestations can include bicuspid aortic valve systolic ejection sound and/or murmur and neurological complaints ${ }^{2}$. Prognosis and survival depend on the disease severity and patient's

a. Dr. Md Nazmul Hasan, Resident, Department of Cardiology, Bangabandhu Sheikh Mujib Medical University, Dhaka, Bangladesh.

b. Prof. Md Abu Siddique, Professor \& Chairman, Department of Cardiology, Bangabandhu Sheikh Mujib Medical University, Dhaka, Bangladesh.

c. Prof. Sajal Krishna Banerjee, Professor, Department of Cardiology, Bangabandhu Sheikh Mujib Medical University, Dhaka, Bangladesh.

d. Dr Abu Sadique Abdullah, Medical Officer, Department of Cardiology, Bangabandhu Sheikh Mujib Medical University, Dhaka, Bangladesh.

Address for Correspondence: Dr Md Nazmul Hasan, Resident, Department of Cardiology, Bangabandhu Sheikh Mujib Medical University, Shahbag, Dhaka, Bangladesh. Phone: +8801675755638, Email: nazmulhasan45cmc@gmail.com

Received: 17 Sep. 2014

Accepted: 7 June 2017 coarctation in a 45-year-old male. Our patient was relatively asymptomatic until he presented with exertional dyspnea and fatigue in his fourth decade of life. The patient was managed by surgery of aorta. After the 6 months follow-up visit, the patient was in good clinical condition.

Keywords: Congenital malformation, aortic coarctation, bicuspid aortic valve, aortic surgery.

(J Bangladesh Coll Phys Surg 2018; 36: 33-36)

age at the time of correction. Death in these patients is usually due to heart failure, coronary artery disease, aortic rupture/dissection, concomitant aortic valve disease, infective endarteritis/endocarditis, or cerebral hemorrhage. There are few reports of patients first diagnosed with uncorrected aortic coarctation at very late age ${ }^{3,4}$. Treatment consists of aggressive hypertension therapy, endocarditis prophylaxis and corrective treatment for coarctation lesions with a high gradient. In this case report, we present aortic coarctation with bicuspid aortic valve in a 45 -year-old male.

\section{Case Report:}

A 45 year-old man was admitted with increasing fatigue and exertional dyspnea. He had been well until 5 months previously. The patient had a medical history of dyslipidemia and hypertension. His hypertension was

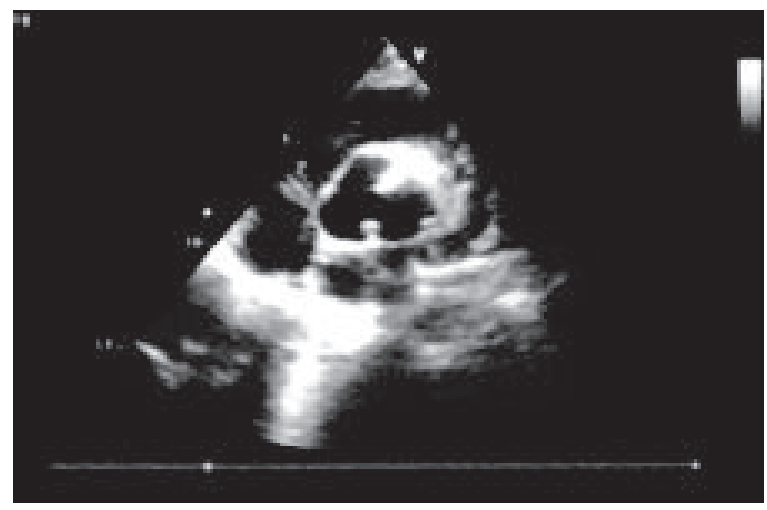

Fig.-1: Transthoracic echocardiography in parasternal short axis view at aortic valve level showing bi-leaflet aortic valve. 
poorly controlled despite a combination of antihypertensive agents (beta-blocker and angiotensin receptor blocker). Physical examination showed blood pressure 140/90 in both arms, a heart rate of 74 beats/ minute and an apical gallop sound (S4) with a continuous murmur over the left parasternal area. There was another systolic murmur over the aortic area. Femoral pulses were palpable bilaterally but weak and delayed compared to the brachial pulses. His echocardiogram showed bicuspid aortic valve with minimal regurgitation, segmental wall motion abnormalities and mild mitral insufficiency and continuous Doppler echocardiography showed a peak systolic gradient of $69 \mathrm{mmHg}$ and a low grade antegrade diastolic flow in the descending thoracic aorta . A cardiac silhouette at the upper limits of normal and notching of the ribs were observed on the chest radiography. Due to the significance of the cardiac dysfunction and his clinical presentation, the patient underwent a cardiac catheterization to evaluate his coronary artery disease. The left ventricular ejection fraction was significantly reduced (Ejection fraction: 30-35\%). There was no evidence of mitral valve prolapse. Computed Tomography (CT Scan) of chest showed a discrete narrowing of the thoracic aorta just distal to the left subclavian artery. Aortography showed a mildly dilated aortic root, minimal aortic valve insufficiency and a significant ring-like stenosis in the thoracic descending aorta. The gradient across the stenosis measured 69 $\mathrm{mmHg}$. The coronary angiography was negative for significant focal coronary artery obstruction. The patient

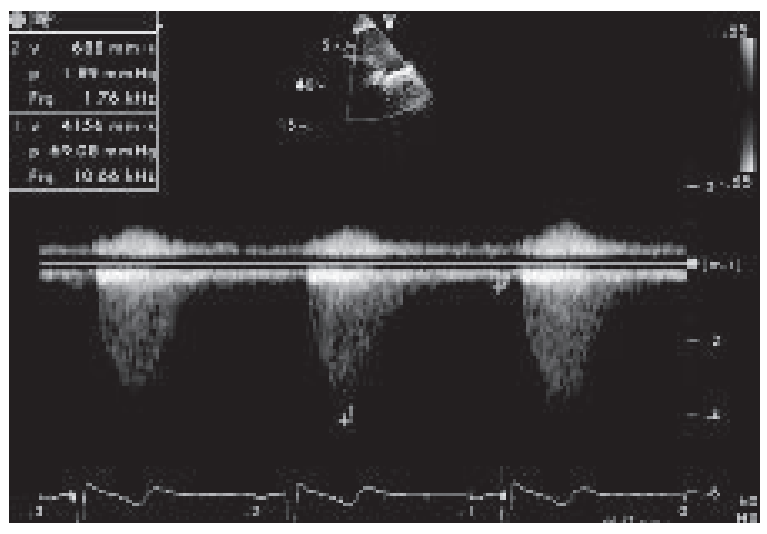

Fig.-2: Continuous Doppler echocardiography showing a peak systolic gradient of $69 \mathrm{mmHg}$ and a low grade antegrade diastolic flow in the descending thoracic aorta (saw tooth). was then referred to cardiothoracic surgery. The procedure was done via left posterolateral thoracotomy from the fifth intercostal space. Since, the collaterals were well recognized before surgery, the procedure was achieved without major bleeding and any adverse event. Furthermore, the patient was adult and any minor
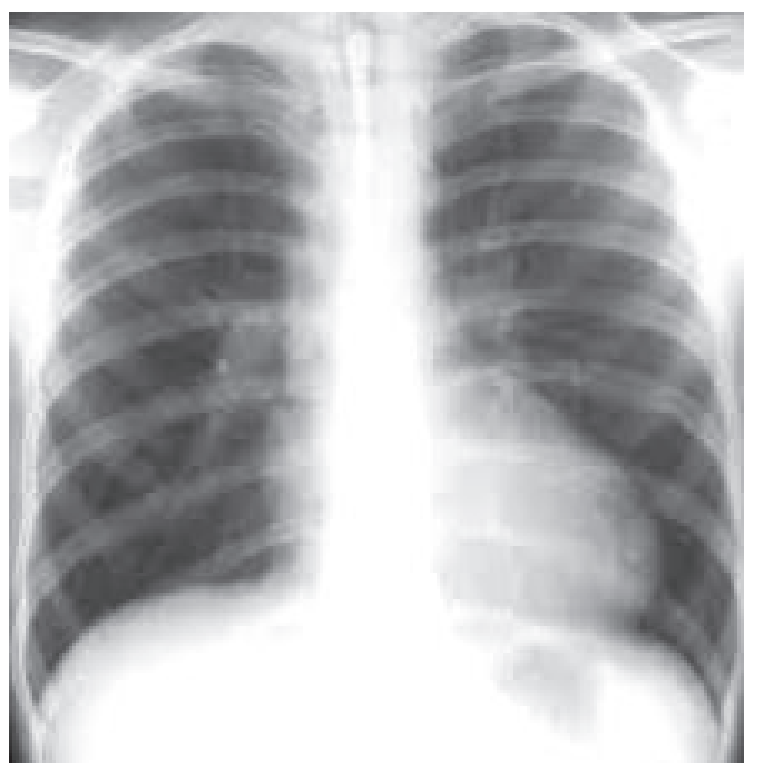

Fig.-3: X-ray chest showing Cardiomegaly with presence of Rib notching in $3^{\text {rd }}$ and $4^{\text {th }}$ rib.

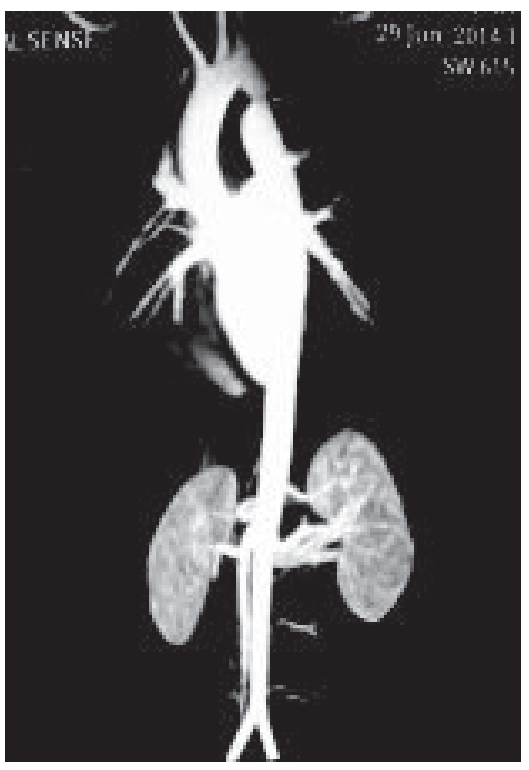

Fig.-4: Computed Tomography (CT Scan) of chest showing a discrete narrowing of the thoracic aorta just distal to the left subclavian artery. 
bleeding has not resulted in requirement of blood transfusion. The coarctated segment was resected totally and end to end anastomosis of thoracic aorta was performed in a standard fashion. The coarctated segment was short in our patient and it was not difficult to get the two ends together without tension on the anastomosis so that we do not considered an interposition graft. The cross clamp time was 26 minutes and because the collaterals were left intact, no malperfusion syndrome occurred. Total hospital stay after procedure was only four days. At the 6 months follow-up visit, the patient was in good clinical condition.

\section{Discussion:}

Coarctation of the aorta $(\mathrm{CoA})$ is typically a discrete narrowing of the thoracic aorta just distal to the left subclavian artery. However, the constriction may be proximal to the left subclavian artery or rarely in the abdominal aorta. In some cases, coarctation presents as a long segment or a tubular hypoplasia. The most frequently associated lesions include bicuspid aortic valve (up to $85 \%$ of the cases), different levels of aortic stenosis, mitral valve stenosis (parachute mitral valve, a complex known as Shone syndrome) ${ }^{5}$. Aortic coarctation presenting during adult life, most frequently represents cases of re-coarctation, following previous transcatheter or surgical therapy, or missed cases of native coarctation. Aortic coarctation may be recognized in the adult, usually because of systemic arterial hypertension and discrepant upper- and lower-extremity pulses. Patients may complain of exertional headaches, leg fatigue or claudication. The reduced life expectancy of patients without correction due to several complications like systemic hypertension, accelerated coronary heart disease, stroke, aortic dissection, and congestive heart failure, demand an early treatment in these patients ${ }^{6}$.

There are different methods employed for the treatment of $\mathrm{CoA}$ in adults, including surgical or percutaneous balloon angioplasty with or without stent placement, and medical therapy.

The 2008 American College of Cardiology/American Heart Association (ACC/AHA) guidelines for adults with congenital heart disease (ACHD), recommend intervention for coarctation in the following circumstances: peak to peak coarctation gradient greater than or equal to $20 \mathrm{mmHg}$, or peak to peak coarctation gradient less than $20 \mathrm{mmHg}$, in the presence of anatomic imaging evidence of significant coarctation with radiologic evidence of significant collateral flow (class IC indication) $)^{7}$.

Surgical repair of coarctation can be achieved by several techniques: resection with end-to-end anastomosis, subclavian flap aortoplasty in infants with long-segment coarctation, a bypass graft across the area of coarctation when the distance to be bridged is too long for an endto-end repair or prosthetic patch aortoplasty ${ }^{8}$. Problems with these techniques have included a significant incidence of aneurysm formation with Dacron patch aortoplasty, and an unacceptably high recoarctation rate with the subclavian flap aortoplasty. The technique of extended end-to-end anastomosis appears to give good short-term to intermediate-term results with a low complication rate and has gained in popularity as the technique of choice when possible to use. A complication associated with all the surgical techniques is aortic dissection, which can occur even late after surgical repair. Surgical mortality is rare (usually less than 1 percent). Morbidity includes early postoperative paradoxical hypertension, left recurrent laryngeal nerve paralysis, phrenic nerve injury, and subclavian steal. Paraplegia due to spinal cord ischemia and mesenteric arteritis with bowel infarction are rare complications ${ }^{9}$.Nowadays, it is generally accepted that these patients require indefinite follow-up by a cardiologist, specialized in the field of congenital heart disease. The frequency in which outpatient visits and tests should take place is highly dependent on the clinical history, the presence of associated cardiac anomalies, type of repair, and the patient's blood pressure. The guidelines recommend that patients who have had surgical repair or percutaneous intervention for coarctation of the aorta should have at least a yearly follow-up and, the evaluation of the coarctation repair site by MRI/CT, should be performed at intervals of 5 years or less, depending on the specific anatomic findings before and after repair (Class I, Level of evidence C). Even if the coarctation repair appears to be satisfactory, late postoperative thoracic aortic imaging should be performed to assess for aortic dilatation or aneurysm formation ${ }^{10}$.

\section{Conclusions:}

The case presented best illustrates that coarctation of the aorta is a congenital cardiac malformation that can 
go undiagnosed until adulthood, having only hypertension as a marker of its presence, because clinical signs can be subtle and overlooked if a complete physical exam is not performed. Nowadays, different surgical and interventional types of treatment are available but this should be individualized for each patient and for each type of coarctation (native coarctation or recoarctation after surgical or interventional treatment).

\section{References:}

1. Grech V. Diagnostic and surgical trends, and epidemiology of coarctation of the aorta in a population-based study. Int $J$ Cardiol. 1999;68:197-202.

2. Cevik S, Izgi C, Cevik C. Asymptomatic severe aortic coarctation in an 80-year-old man. Tex Heart Inst J. 2004;31:429-431.

3. Warnes CA, Deanfield JE. Congenital heart disease in adults. In: (ed.) Alexander RW. et al. Hurst's The Heart Volume 2, 11 th edition. New York: McGraw Hill Professional. 2004:1866.

4. Campbell M. Natural history of coarctation of the aorta. Br Heart J. 1970;32:633-640.
5. Jenkins NP, Ward AR. Coarctation of the aorta: natural history and outcome after surgical treatment. QJM. 1999;92:365-371.

6. Anagnaostopoulos-Tzifa A, Management of Aortic Coarctation in Adults: Endovascular Versus Surgical Therapy. Hellenic $J$ Cardiol 48: 290-295, 2007.

7. Warnes CA, Williams RG, Bashore TM, et al. ACC/AHA 2008 Guidelines for the Management of Adults with Congenital Heart Disease: a report of the American College of Cardiology/ American Heart Association Task Force on Practice Guidelines (writing committee to develop guidelines on the management of adults with congenital heart disease). Circulation 2008; 118:e714-833.

8. Horvath R, Towgood A, Sandhu SK. Role of Transcatheter Therapy in the Treatment of Coarctation of the Aorta. J Invasive Cardiol 2008; 20(12):660-663.

9. Manganas C, Iliopoulos J, Chard RB, Nunn GR. Reoperation and coarctation of the aorta: the need for lifelong surveillance. Ann Thorac Surg 2001;72:1222 -1224.

10. Vriend JWJ, Mulder BJM. Late complications in patients after repair of aortic coarctation: implications for management. Int J Cardiol 2005, 101:399- 406. 